\title{
Modelling for the Reduction and Spread Transmission of the Dynamics and Control of Zika virus Disease: Using Ordinary Differential Equations
}

\author{
*1ADAMU, G; ${ }^{2}$ NDAGARA, AS; ${ }^{2}$ MAMUDA, M; ${ }^{3}$ OMATOLA, IU \\ ${ }^{I}$ Department of Mathematics, University of Ilorin, Nigeria \\ ${ }^{2}$ Department of Mathematics, Niger State College of Education, Minna, Nigeria \\ ${ }^{3}$ Department of Mathematic, Kogi State University Ayingba, Nigeria \\ *Corresponding AuthorEmail; ahlul_baity@yahoo.com
}

\begin{abstract}
The Zika virus is a flavivirus which upon infection in humans causes an illness known as Zika fever. It is commonly identified by popular rash, mild fever and arthritis. The Zika virus (ZIKV) was first identified and isolated from the Zika forest in Uganda in 1947. This paper focuses on human recovery from treatment and death of mosquitoes from insecticides in order to reduce the spread of the virus. This shows that the ZIKV is endemic in a population when the illness exists and is a threat to a population if there is no control. Therefore, the model formulated is unique for analysis. The result of this study shows that the treatment technique without insecticides has no significant reduction in the infected vectors and hosts. The stability of the disease shows stable with $R_{o}>1$. Finally, the best option is to use an insecticide to reduce the spread of the virus on both infected hosts and vectors.
\end{abstract}

\section{DOI:https://dx.doi.org/10.4314/jasem.v23i12.3}

Copyright: Copyright (C) 2019 Adamu et al. This is an open access article distributed under the Creative Commons Attribution License (CCL), which permits unrestricted use, distribution, and reproduction in any medium, provided the original work is properly cited.

Dates: Received: 19 October 2019; Revised: 24 November 2019; Accepted: 20 December 2019

Keywords: Zika Virus, Modelling, Transmission, Dynamics and Analysis

Zika virus ZIKV is a mosquito-borne flavivirus disease that was first identified in monkeys in Uganda in 1947 through a network that monitored yellow fever. It was later identified in humans in 1952 in Uganda and Tanzania. The outbreaks of Zika virus disease has been recorded in Africa, America, Asia and the Pacific. From the 1960s to 1980 s, human infections were found across Africa and Asia, typically accompanied by mild illness (Dick, 1952). The first large outbreak of disease caused by Zika infection was reported from the Island of Yap (Federated States of Micronesia) in 2007. In July 2015, Brazil reported an association between Zika Virus infection and Guillain-Barré syndrome. In October 2015, Brazil reported an association between Zika Virus infection and microcephaly (WHO, 2016). Zika Virus is primarily transmitted to people through the bite of an infected mosquito from the Aedes genus, mainly Aedes egypti in tropical regions. Aedes mosquitoes usually bite during the day, peaking during early morning and late afternoon/evening. This is the same mosquito that transmits dengue, chikungunya and yellow fever. Sexual transmission of Zika Virus is also possible. Other modes of transmissions such as blood transfusion are being investigated (Musso, 2015). The incubation period of Zika Virus disease is not clear but is likely to be a few days. The symptoms are similar to other infections such as dengue, fever, skin rashes, conjunctivitis, muscle and joint pain, malaise, and headache. These symptoms are usually mild and last for 2-7 days (WHO, 2016).
ZIKV was first isolated in 1947 from a febrile sentinel rhesus monkey in the Zika forest, a research station of the East African Virus Research Institute now Uganda Virus Research Institute in Entebbe, Uganda (Dick, 1952a, Dick and Haddow 1952b). The virus was isolated subsequently from Aedes Africanus mosquitoes in the same forest (Dick, 1952, McCrae and Kirya, 1982). The multiple monkey species in the Zika forest were found to be seropositive for ZIKV (McCrae and Kirya, 1982). Small mammals in the Zika forest including squirrels, tree rats, giant pouched ratsand civets did not show serological evidence of ZIKV infection, consistent with a model where primates (both humans and monkeys) are the primary vertebrate hosts for ZIKV (Haddow et al., 1964). Multiple species of Aedes mosquitoes contribute to enzootic maintenance of ZIKV, but likely only a subset of these transmits the virus to humans (Diagne, et al., 2015, Diallo et al., 2014). In the case of the West African Ebola outbreak of 2014 to present, rapid communication, adoption of effective outbreak tracing and control measures and cultural changes reduced transmission to the point that vaccine trial efficacy endpoints could not be met. Yap Island in Micronesia, the overall attack rate observed for confirmed and probable Zika Virus disease among patients presenting to health care facilities was 14.6 per 1000 Yap residents range of 3.6 to 21.5 per 1000 population. During what appears to have been a four-month, selflimited outbreak, it is estimated that $73 \%$ of Yap residents (3 years of age or older) were infected with a Zika Virus strain hypothesized to have been brought 
to the island by an imported non-human primate (Musso, et al., 2014). This suggests that, with the current Zika outbreak, the virus may spread so efficiently that by the time a vaccine becomes available to test in human clinical trials and identifying large naïve at risk, population may be an obstacle to demonstrating efficacy.

ZikaVirus evolution and spread is constrained by both human and insect hosts, and this creates an opportunity to develop countermeasure strategies focusing on either or both. The interaction between pathogen and host biology will impact the incidence, prevalence and eventual distribution of the virus. As Zika adapts to new niches in the Americas, the roles played by humans and non-human primates, other animals and arthropods as primary and intermediate hosts must be understood. Factors which will influence the rate of spread include availability of vector species, temperature and humidity available to support transmissibility, and high mosquito to human contact rates. It is similar to dengue and chikungunya, Aedes egypti and albopictus appear to be the leading candidate Zika vectors in the outbreak. Potential involvements of other insect vectors including Culex Sp. mosquitoes are currently being examined (Mlakaret al., 2016, Oliveira, et al., 2016). In the outbreak on Yap Island, 12 mosquito species belonging to four generation were identified as potential vectors and hensilli. Farmer was the predominant vector species (Musso, et al., 2014). The symptoms are similar to other infections such as dengue, fever, skin rashes, conjunctivitis, muscle and joint pain, malaise and headache. These symptoms are usually mild and last for 2-7 days. The recent ZIKV incidence in Brazil and French Polynesia possibly compels World Health Organization WHO to declare a Public Health Emergency of International Concern in response to the clusters of microcephaly and other neurological disorders. Apart from the major outbreak in French Polynesia, ZIKV incident shows 42 Guillain-Barre syndrome cases between March 2014 and May 2015 in the same region and severe brain lesions were reported (Ebenezer and Kazeem, 2016). The current outbreak of ZIKV in Cape Verde is showing that ZIKV is now returning to its place of origin. The presence of Aedes mosquito species in Africa which has caused several outbreaks of dengue fever in Africa including the 2007 outbreak in Gabon, 2010 outbreak in Kenya and 2013 outbreak in the United Republic of Tanzania suggest the possibility of re-emergence of ZIKV in Africa. The potential transmission of ZIKV to human beings is evident. While ZIKV is currently endemic in some parts of the world, a little is known by many people in Africa. Little or no effort is being made to understand the dynamics of ZIKV infection to human, the modes of transmission and possible ways of prevention or control mechanisms. That is to say, the disease has been neglected in Africa. Modelling the dynamics of ZIKV will help bring awareness to people and have a better understanding of the transmission dynamics of $\mathrm{ZIKV}$, the risk associated to the disease, and measures to control the disease (Mpeshe, et al., 2017). Consequently, at this time there were no recognized vaccines or drugs to cure the ZIKV infection.

A lot of mathematical models have been built as tools for investigating the dynamics transmission of infectious diseases. Ebenezer and Kazeem (2016) formulated a mathematical model for optimal control strategy of Zika Virus to examine the effect of mass treatment and insecticide, in order to minimize the number of Zika infected hosts and vectors with optimal cost of mass treatment and insecticide applying Pontryagin's maximum principle. The simulation results were illustrating the population dynamics with prevention and treatment as controls. In their models, death due to infections was ignored and all recruitment rates were susceptible. Gao, et al., (2016) built a mathematical model to investigate the impact of mosquito-borne and sexual transmission on the spread and control of ZIKV and standardized the model to ZIKV contagion. They suggested that the Prevention and the control efforts against ZIKV should target both the mosquito-borne and sexual transmission. Adams et al., (2017) developed a mathematical model to simulate vector-borne transmission for people and mosquitoes. The model incorporated delays as a result of the intrinsic (human) and extrinsic (vector) incubation periods. Onuorah et al., (2016) developed a deterministic model for the dynamics of Zika Virus. The model incorporated vital dynamics for both the human and vector compartments. Jiraporn (2017) formulated a transmission model of Zika Virus between two population, human and mosquito to find the steady states of our equations. The standard dynamical modelling method is used for analysis of model, a disease free steady state and an endemic steady state. Their model did not specify the actual dynamics of the infectious disease. Mpeshe etal.,(2017) developed a mathematical model for the dynamics of Zika Virus fever. Using sensitivity analysis to indicate that $R_{0}=$ $7.03>1$ is most sensitive to the natural death rate of Aedes mosquitoes, and least sensitive to the recovery rate of human. This means that increasing the natural death rate of mosquitoes and the recovery rate of human will reduce $R_{0}$ and hence, control of the disease is possible. Their result does not incorporate the effect of Zika Virus fever (ZIKVF) in the society. 
Adamu etal.,(2017)developed a deterministic mathematical model of Zika virus using ordinary differential equations with two control strategies: treatment for humans and insecticide spray for mosquitoes. Homotopy Perturbation Method (HPM) was used to obtain the approximate solution of the model. From their model, 59\% effective administration of insecticide spray proved effective which showed a great reduction in the infected humans as well as infected vector population.

Incubation period for the virus is suggested to be 3-12 days while the course of infection may extend up to 27 days which is mostly self-limiting. Therefore, there is need to develop a mathematical model to control the spread of ZIKV. This study aims to develop and analyze a model of non-linear ordinary differential equations.

The aim of this paper is to develop a model for the dynamics and control of Zika Virus in order to reduce the spread of transmission among the public using ordinary differential equations.

The Model Formulation: In this research work, the instrument use to investigate the model formulated is a deterministic method of ODE using MAPLE 18 software for analysis. Similarly, the model is an extension of Adamu et al.,(2017). This model analyse the basic reproduction number, endemic state and global stability in order to reduce the threat in a population and awareness. While Adamu et al., (2017) developed a model from an existing model of Ebenezer and Kazeem, (2016) and employed HPM to analyse the error.

Model Description: In this paper, the population of interest is divided into human host and vector. Susceptible individuals are recruited into the population by input rate $\Lambda$. When susceptible individuals have contact(s) with some infected vectors, the probability is $\beta_{h}$, but it did not make infectious except with a proportion of infected vectors. $\beta_{v}$ is the spread rate from mosquitoes to human while $\lambda$ is the probability of infectious human being recovered, this means some individuals move to recovered classdue to insecticide of vectors and treatment host with rates $\delta_{h}$ and $\delta_{v}$ respectively. Finally, $\mu$ is the parameter that represents natural death in each classes while $S_{h}$ represents susceptible human, $S_{v}$ is a susceptible vector, $I_{h}$ infectious human, $I_{v}$ infectious vectors, $R_{h}$ is a recovered human, $\mu_{h}$ natural death rate of human, $\mu_{v}$ natural death of vector, $\delta_{v}$ represents death rate from insecticide, $\delta_{h}$ represents recovery rate from treatment (both are control strategies respectively), $\beta_{h}$ the spread rate from humans to mosquitoes, $\beta_{v}$ spread rate from mosquitoes to humans, $\wedge_{h}$ is the recruitment number of susceptible humans, $\wedge_{v}$ recruitment number of susceptible vector, $\mu_{0}$ death due to infectious, $\lambda$ probability of infectious humans being recovered.

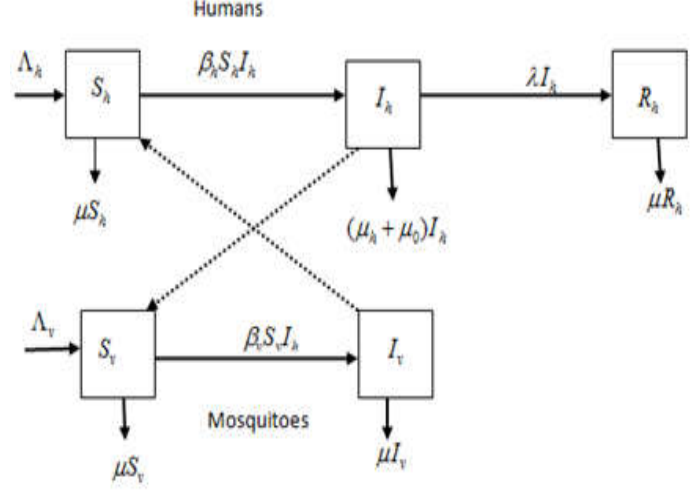

The Model Transmission of a Zika Virus

The positions of ordinary differential equations related to the exceeding model diagram are:

$$
\begin{aligned}
& \frac{d S_{h}}{d t}=\wedge_{h}-\mu_{h} S_{h}-\beta_{h} S_{h} I_{v}(1) \\
& \frac{d I_{h}}{d t}=\beta_{h} S_{h} I_{v}-\left(\mu_{h}+\mu_{0}+\lambda+\delta_{h}\right) I_{h}(2) \\
& \frac{d R_{h}}{d t}=\left(\lambda+\delta_{h}\right) I_{h}-\mu_{h} R_{h} \\
& \frac{d S_{v}}{d t}=\wedge_{v}-\beta_{v} S_{v} I_{h}-\left(\mu_{v}+\delta_{v}\right) S_{v} \\
& \frac{d I_{v}}{d t}=\beta_{v} S_{v} I_{h}-\left(\mu_{v}+\delta_{v}\right) I_{v}
\end{aligned}
$$

\section{Positivity of Solution}

Theorem 1 :Let the set

$\left\{S_{h}(0)>0, S_{V}(0)>0,\left(I_{h}(0), R_{h}(0), I_{V}(0) \geq 0\right)\right\} \in D$

Then the solution set $\left\{S_{h}, I_{h}, R_{h}, S_{V}, I_{V}\right\}(t)$ of the system of equations (1-5) is positive for all $t>0$ (Cooke and Van Den Driessche, 1996). 
Proof: From the first equation (1), give

$\frac{d S_{h}}{d t}=\wedge_{h}-\mu_{h} S_{h}-\beta_{h} S_{h} I_{v} \geq-\left(\mu_{h}+\beta_{h} I_{v}\right) S_{h}$

(6)

$\frac{d S_{h}}{d t}=-\left(\mu_{h}+\beta_{h} I_{v}\right) S_{h}$

Separating the variables and integrating we have; $S_{h}(t) \geq A e^{-\left(\mu_{h}+\beta_{h} I_{v}\right) t}$

Where $A=e^{C}$

Using the initial condition $t=0 \Rightarrow S_{h}(0) \geq A$

Therefore,

$S_{h}(t) \geq S_{h}(0) e^{-\left(\mu_{h}+\beta_{h} I_{v}\right) t} \geq 0$

From equation (2)

$\left.\frac{d I_{h}}{d t}=\beta_{h} S_{h} I_{v}-\left(\mu_{h}+\mu_{0}+\lambda+\delta_{h}\right) I_{h} \geq-\left(\mu_{h}+\mu_{0}+\lambda+S_{h}, I_{h}^{h}\right) R_{h}, S_{V}, I_{V}\right)=\left[\frac{\Lambda_{h}}{\mu_{h}}, 0,0, \frac{\Lambda_{V}}{\mu_{V}}, 0\right]$

$\frac{d I_{h}}{d t} \geq-\left(\mu_{h}+\mu_{0}+\lambda+\delta_{h}\right) I_{h}$

Endemic Equilibrium State of the Model: The endemic equiulibrum state of our model equations (1-5) is given bellow:

(11)

$$
\begin{gathered}
S_{h}^{* *}=\frac{\left(\mu_{V}+\delta_{V}\right) \Lambda_{h}-\left(\mu_{h}+\mu_{0}+\lambda+\delta_{h}\right) \Lambda_{V}}{\left(\mu_{V}+\delta_{V}\right) \mu_{h}-\left(\mu_{h}+\mu_{0}+\lambda+\delta_{h}\right)\left(\mu_{V}+\delta_{V}\right)} \\
\left.I_{h}^{* *}=\frac{\frac{\mu\left(\left(\mu_{V}+\delta_{V}\right) \Lambda_{h}-\left(\mu_{h}+\mu_{0}+\lambda+\delta_{h}\right) \Lambda_{V}\right)}{\left(\mu_{V}+\delta_{V}\right) \mu_{h}-\left(\mu_{h}+\mu_{0}+\lambda+\delta_{h}\right)\left(\mu_{V}+\delta_{V}\right)}-\Lambda_{h}}{\left(\mu_{h}+\mu_{0}+\lambda+\delta_{h}\right)}\right) \\
R_{h}^{* *}=\frac{(16)}{\left(\lambda+\delta_{h}\right)\left(\frac{\mu\left(\left(\mu_{V}+\delta_{V}\right) \Lambda_{h}-\left(\mu_{h}+\mu_{0}+\lambda+\delta_{h}\right) \Lambda_{V}\right)}{\left(\mu_{V}+\delta_{V}\right) \mu_{h}-\left(\mu_{h}+\mu_{0}+\lambda+\delta_{h}\right)\left(\mu_{V}+\delta_{V}\right)}-\Lambda_{h}\right)} \\
I_{V}^{* *}=\frac{\beta_{V} W}{\left(\mu_{V}+\delta_{V}\right)}
\end{gathered}
$$

Where

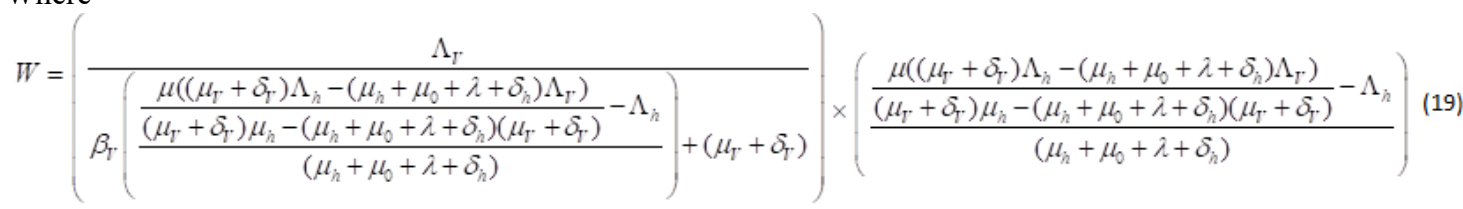


Basic Reproduction Number, $R_{0}$ : In a more complicated epidemic, the compute $R_{0}$ used the next generation operator approach by (Van Den Driessche, 2017)..From the system equation (1-5) we define

$f_{i}$ and $v_{i}$ as;

$$
\begin{gathered}
f_{i}=\left[\begin{array}{c}
\beta_{h} S_{h} I_{V} \\
\beta_{V} S_{V} I_{h}
\end{array}\right] \text { and } v_{i}=\left[\begin{array}{c}
\left(\mu_{h}+\mu_{0}+\lambda+\delta_{h}\right) I_{h} \\
\left(\mu_{V}+\delta_{V}\right) I_{V}
\end{array}\right](20) \\
F V^{-1}=\left[\frac{\partial f_{i}\left(E_{0}\right)}{\partial x_{j}}\right]\left[\frac{\partial v_{i}\left(E_{0}\right)}{\partial x_{j}}\right]^{-1} \\
=\left[\begin{array}{cc}
0 & \beta_{h} S_{h} \\
\beta_{V} S_{V} & 0
\end{array}\right]\left[\begin{array}{cc}
\frac{1}{\left(\mu_{0}+\mu_{h}+\lambda+\delta_{h}\right)} & 0 \\
0 & \frac{1}{\left(\mu_{V}+\delta_{V}\right)}
\end{array}\right]=\left[\begin{array}{cc}
0 & \frac{\beta_{h} S_{h}}{\left(\mu_{V}+\delta_{V}\right)} \\
\frac{\beta_{V} S_{V}}{\left(\mu_{0}+\mu_{h}+\lambda+\delta_{h}\right)} & 0
\end{array}\right]
\end{gathered}
$$

Therefore, the basic reproduction number $R_{0}=\rho\left(F V^{-1}\right)=$ spectra radius of $F V^{-1}$

Hence

$$
\begin{aligned}
& R_{0}=\sqrt{\frac{\left(\mu_{v}+\delta_{v}\right)\left(\mu_{0}+\mu_{h}+\lambda+\delta_{h}\right) \beta_{h} S_{h} \beta_{V} S_{V}}{\left(\mu_{0}+\mu_{h}+\lambda+\delta_{h}\right)\left(\mu_{V}+\delta_{V}\right)}} \\
& R_{0}=\sqrt{\frac{\left(\mu_{v}+\delta_{v}\right)\left(\mu_{0}+\mu_{h}+\lambda+\delta_{h}\right) \beta_{h}\left(\frac{\Lambda_{h}}{\mu_{h}}\right)_{h} \beta_{V}\left(\frac{\Lambda_{V}}{\mu_{V}}\right)}{\left(\mu_{0}+\mu_{h}+\lambda+\delta_{h}\right)\left(\mu_{V}+\delta_{v}\right)}}
\end{aligned}
$$

Global Stability of Disease Free Equilibrium State (DFE): Theorem 2: The disease free equilibrium state is globally asymptotically stable if $R_{0}<1$

Proof: To establish the global stability of disease-free equilibrium, the two conditions (H1) and (H2) as in (Okongo, 2015) must be satisfied for $R_{0}<1$. The model equations (1-5) are in the form of

$$
\begin{aligned}
& \frac{d X_{1}}{d t}=F\left(X_{1}, X_{2}\right)(24) \\
& \frac{d X_{2}}{d t}=G\left(X_{1}, X_{2}\right) ; G\left(X_{1}, 0\right)=0
\end{aligned}
$$

Where $X_{1}=\left(S_{h}{ }^{0}, R_{h}{ }^{0}, S_{V}{ }^{0}\right), X_{2}=\left(I_{h}{ }^{0}, I_{V}{ }^{0}\right)$ with the compartments of $X_{1} \in \square^{3}$ denoting the uninfected population and the compartments of $X_{2} \in \square^{2}$ denoting the infected population
Now, the DFE is denoted as

$$
E^{0}=\left(X^{*}, 0\right)(26)
$$

Where

$$
X_{1}^{*}=\left(N^{0}, 0\right)(27)
$$

Now, for the first condition, that is globally asymptotically stable of $X_{1}^{*}$, gives

$$
\frac{d X_{1}}{d t}=F\left(X_{1}, 0\right)=\left[\begin{array}{l}
\wedge_{h}-\mu_{h} S_{h}^{0} \\
-\mu_{h} R_{h}^{0} \\
\wedge_{r}-\left(\mu_{r}+\delta_{v}\right) S_{V}{ }^{0}
\end{array}\right]
$$

Solving, gives

$$
S_{h}^{0}(t)=\frac{\Lambda_{h}}{\mu_{h}}-\frac{\Lambda_{h}}{\mu_{h}} e^{-\mu t}+S_{h}^{0}(0) e^{-\mu t}
$$




$$
\begin{aligned}
& R_{h}^{0}(t)=R_{h}^{0}(0) e^{-\mu t}(30) \\
& S_{V}^{0}(t)=\frac{\Lambda_{V}}{\mu_{V}}-\frac{\Lambda_{V}}{\mu_{V}} e^{-\mu t}+S_{V}^{0}(0) e^{-\mu t}
\end{aligned}
$$

Clearly, from equation (22) gives

$S_{h}{ }^{0}(t)+R_{h}^{0}(t)+S_{V}^{0}(t) \rightarrow N^{0}(t)$ as $t \rightarrow \infty$, regardless of the values o $S_{h}^{0}(t), R_{h}{ }^{0}(t)$ and $S_{V}{ }^{0}(t)$. Hence, DFE is GAS .Next for the second condition,

$$
\begin{aligned}
& A=\left(\begin{array}{cc}
-\left(\mu_{0}+\mu_{h}+\lambda+\delta_{h}\right) & \beta_{h} S_{h}^{*} \\
\beta_{V} S_{V}^{*} & -\left(\mu_{V}+\delta_{V}\right)
\end{array}\right) \\
& G=\left(\begin{array}{cc}
-\left(\mu_{0}+\mu_{h}+\lambda+\delta_{h}\right) & \beta_{h} S_{h} \\
\beta_{V} S_{V} & -\left(\mu_{V}+\delta_{V}\right)
\end{array}\right)
\end{aligned}
$$

We need to show that $\left(\mathrm{H}_{2}\right)$ holds in the system (1 5)

$$
G\left(X_{1}, X_{2}\right)=A Z-G\left(X_{1}, X_{2}\right)
$$

$$
\begin{gathered}
G=\left(\begin{array}{cc}
-\left(\mu_{0}+\mu_{h}+\lambda+\delta_{h}\right) & \beta_{h} S_{h}^{*} \\
\beta_{V} S_{V}^{*} & -\left(\mu_{V}+\delta_{V}\right)
\end{array}\right)\left(\begin{array}{l}
I_{h} \\
I_{V}
\end{array}\right)-\left(\begin{array}{cc}
-\left(\mu_{0}+\mu_{h}+\lambda+\delta_{h}\right) & \beta_{h} S_{h} \\
\beta_{V} S_{V} & -\left(\mu_{V}+\delta_{V}\right)
\end{array}\right)\left(\begin{array}{l}
I_{h} \\
I_{V}
\end{array}\right) . \\
G=\left(\begin{array}{cc}
0 & \beta_{V}\left(S_{V}^{*}-S_{V}\right) \\
\beta_{V}\left(S_{V}^{*}-S_{V}\right) & 0
\end{array}\right)\left(\begin{array}{l}
I_{h} \\
I_{V}
\end{array}\right)=\left(\begin{array}{l}
\beta_{V}\left(S_{V}^{*}-S_{V}\right) I_{V} \\
\beta_{V}\left(S_{V}^{*}-S_{V}\right) I_{h}
\end{array}\right)(36)
\end{gathered}
$$

Since $S_{h}{ }^{*} \geq S_{h}$ and $S_{V}^{*} \geq S_{V}$ then

$\stackrel{\Lambda}{G} \geq 0$. Hence, $\left(H_{1}\right)$ and $\left(H_{2}\right)$ are satisfied.

Therefore the DFE state is locally asymptotically stable provided $R_{0}<1$.
Global Stability Analysis of Endemic Equilibrium State (DFE): Theorem 3: The endemic equilibrium point is globally asymptotically stable if $R_{0}>1$

Proof: Now, consider the lyapunov function, $\mathrm{L}$ for equations (1-5) as

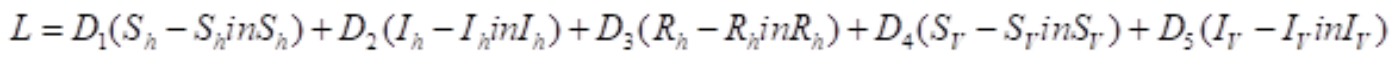

Where,

$D_{1}, D_{2}, D_{3}, D_{4}$ and $D_{5}$ are positive constants

$$
\begin{aligned}
& \Lambda_{h}=\left(\beta_{h} I_{V}^{*}+\mu_{h}\right) S_{h}{ }^{*}, \beta_{h}=\frac{\left(\mu_{h}+\mu+\lambda+\delta_{h}\right) I_{h}{ }^{*}}{S_{h}^{*} I_{V}{ }^{*}},\left(\lambda+\delta_{h}\right)=\frac{\mu R_{h}{ }^{*}}{I_{h}{ }^{*}}, \\
& \Lambda_{V}=\left(\beta_{V} I_{h}{ }^{*}+\mu_{V}+\delta_{V}\right) S_{V}{ }^{*}, \beta_{V}=\frac{\left(\mu_{h}+\delta_{V}\right) I_{V}{ }^{*}}{S_{V}{ }^{*} I_{h}{ }^{*}}
\end{aligned}
$$

Differentiating lyapunov function with respect to time gives:

$$
\frac{d L}{d t}=D_{1}\left(1-\frac{S_{h}^{*}}{S_{h}}\right) \frac{d S_{h}}{d t}+D_{2}\left(1-\frac{I_{h}^{*}}{I_{h}}\right) \frac{d I_{h}}{d t}+D_{3}\left(1-\frac{R_{h}^{*}}{R_{h}}\right) \frac{d R_{h}}{d t}+D_{4}\left(1-\frac{S_{V}^{*}}{S_{V}^{*}}\right) \frac{d S_{V}}{d t}+D_{5}\left(1-\frac{I_{V}^{*}}{I_{V}}\right) \frac{d I_{V}}{d t}
$$




$$
\begin{aligned}
& \frac{d L}{d t}=D_{1}\left(1-\frac{S_{h}^{*}}{S_{h}}\right)\left(\wedge_{h}-\mu_{h} S_{h}-\beta_{h} S_{h} I_{v}\right)+D_{2}\left(1-\frac{I_{h}^{*}}{I_{h}}\right)\left(\beta_{h} S_{h} I_{v}-\left(\mu_{h}+\mu_{0}+\lambda+\delta_{h}\right) I_{h}\right) \\
& +D_{3}\left(1-\frac{R_{h}^{*}}{R_{h}}\right)\left(\left(\lambda+\delta_{h}\right) I_{h}-\mu_{h} R_{h}\right)+D_{4}\left(1-\frac{S_{V}^{*}}{S_{V}}\right)\left(\wedge_{v}-\beta_{v} S_{v} I_{h}-\left(\mu_{v}+\delta_{v}\right) S_{v}\right) \\
& +D_{5}\left(1-\frac{I_{V}^{*}}{I_{V}}\right)\left(\beta_{v} S_{v} I_{h}-\left(\mu_{v}+\delta_{v}\right) I_{v}\right) \\
& \frac{d L}{d t}=D_{1}\left(1-\frac{S_{h}^{*}}{S_{h}}\right)\left(\wedge_{h}-\left(\mu_{h}+\beta_{h} I_{v}\right) S_{h}\right)+D_{2}\left(1-\frac{I_{h}^{*}}{I_{h}}\right)\left(\beta_{h}-\frac{\left.\left(\mu_{h}+\mu_{0}+\lambda+\delta_{h}\right) I_{h}\right)}{S_{h} I_{v}}\right) \\
& +D_{3}\left(1-\frac{R_{h}^{*}}{R_{h}}\right)\left(\left(\lambda+\delta_{h}\right)-\frac{\mu_{h} R_{h}}{I_{h}}\right)+D_{4}\left(1-\frac{S_{V}^{*}}{S_{V}}\right)\left(\wedge_{v}-\left(\beta_{v} I_{h}+\left(\mu_{v}+\delta_{v}\right)\right) S_{v}\right) \\
& +D_{5}\left(1-\frac{I_{V^{*}}^{*}}{I_{r}}\right)\left(\beta_{v}-\frac{\left(\mu_{v}+\delta_{v}\right) I_{v}}{S_{v} I_{h}}\right) \\
& \frac{d L}{d t}=D_{1}\left(1-\frac{S_{h}^{*}}{S_{h}^{*}}\right)\left(\left(\beta_{h} I_{V}^{*}+\mu_{h}\right) S_{h}^{*}-\left(\mu_{h}+\beta_{h} I_{v}\right) S_{h}\right) \\
& +D_{2}\left(1-\frac{I_{h}^{*}}{I_{h}}\right)\left(\frac{\left(\mu_{h}+\mu^{*}+\lambda+\delta_{h}\right) I_{h}^{*}}{S_{h}^{*} I_{V}^{*}}-\frac{\left(\mu_{h}+\mu_{0}+\lambda+\delta_{h}\right) I_{h}}{S_{h} I_{v}}\right) \\
& +D_{3}\left(1-\frac{R_{h}^{*}}{R_{h}^{*}}\right)\left(\frac{\mu R_{h}^{*}}{I_{h}^{*}}-\frac{\mu_{h} R_{h}}{I_{h}}\right)+D_{4}\left(1-\frac{S_{V}^{*}}{S_{V}^{*}}\right)\left(\left(\beta_{V} I_{h}^{*}+\mu_{V}+\delta_{V}\right) S_{V}^{*}-\left(\beta_{v} I_{h}+\left(\mu_{v}+\delta_{v}\right)\right) S_{v}\right) \\
& +D_{5}\left(1-\frac{I_{V}^{*}}{I_{V}}\right)\left(\frac{\left(\mu_{h}+\delta_{V}\right) I_{V}^{*}}{S_{V}^{*} I_{h}^{*}}-\frac{\left(\mu_{v}+\delta_{v}\right) I_{v}}{S_{v} I_{h}}\right)
\end{aligned}
$$

Therefore, $\frac{d L}{d t}=0$

$$
S_{h}=S_{h}{ }^{*}, I_{h}=I_{h}{ }^{*}, R_{h}=R_{h}{ }^{*}, S_{V}=S_{V}{ }^{*}, I_{V}=I_{V}{ }^{*} \text { and } \frac{d L}{d t}<0 \text { for } S_{h}, I_{h}, R_{h}, S_{V}, I_{V}>0
$$

Hence if $R_{0}>1$ then, the model equation (1-5) has a unique endemic point which is globally asymptotically stable.

Numerical Simulations: The numerical simulations were carried out using the below table that described the parameters, variables and source, with the help of MAPLE 18 software.

\section{DISCUSSION}

The model formulated focuses on treatment human and spray of insecticides on vectors to reduce the risk of virus in the society. Insecticides on vectors should also be put in place where the cases of suspected individual are reported. The graph in Fig. 1 shows 50\% effectively treatment for human in sixty days which reduced the infectious when the rate of treatment on human forced the virus to decrease. This can also be observed from the graph in Fig 2 on infected vector when the rate of spray insecticide on vectors is effective. The infected mosquitoes will also decrease which shows the importance of control strategies.

Moreover, when the rates of $\delta_{h}$ and $\delta_{v}$ are either increased or decreased the situation will change either the virus to persist or die out in a community depend of the control strategy employed. Finally, the graph in Fig 3 shows the significance of control on recovered class where individuals are all recovered at a very high rate. This can be observed from the graph which shows the effectiveness of the control strategies in sixty days. 
Table 1: Description on Variables, Parameters and their Sources

\begin{tabular}{|c|c|c|c|}
\hline $\mathrm{S} / \mathrm{N}$ & Variable & Value & Source \\
\hline 1. & $S_{h}(t)$ & 0.8 & Mpeshe, et al., (2017) \\
\hline 2. & $S_{V}(t)$ & 0.8 & “ \\
\hline 3. & $I_{h}(t)$ & 0.2 & “ \\
\hline 4. & $I_{v}(t)$ & 0.1 & “ \\
\hline 5. & $R_{h}(t)$ & 0.2 & Mpeshe, et al., (2017) \\
\hline 6. & $\beta_{h}$ & 0.5 & Gao, et al., (2016) \\
\hline 7. & $\beta_{v}$ & 0.4 & Gao, et al., (2016) \\
\hline 8. & $\lambda$ & 0.0033 & Assumed \\
\hline 9. & $\mu_{0}$ & 0.5 & Gao, et al., (2016) \\
\hline 10. & $\mu_{h}$ & 0.0027 & Mpeshe, et al., (2017) \\
\hline 11. & $\mu_{v}$ & 0.0714 & Mpeshe, et al., (2017) \\
\hline 12. & $\delta_{v}$ & 0.56 & Assumed \\
\hline 13. & $\delta_{h}$ & 0.05 & Gao, et al., (2016) \\
\hline 14. & $\Lambda_{h}$ & 0.0000001 & Assumed \\
\hline 15. & $\Lambda_{v}$ & 0.071 & Gao, et al., (2016) \\
\hline
\end{tabular}

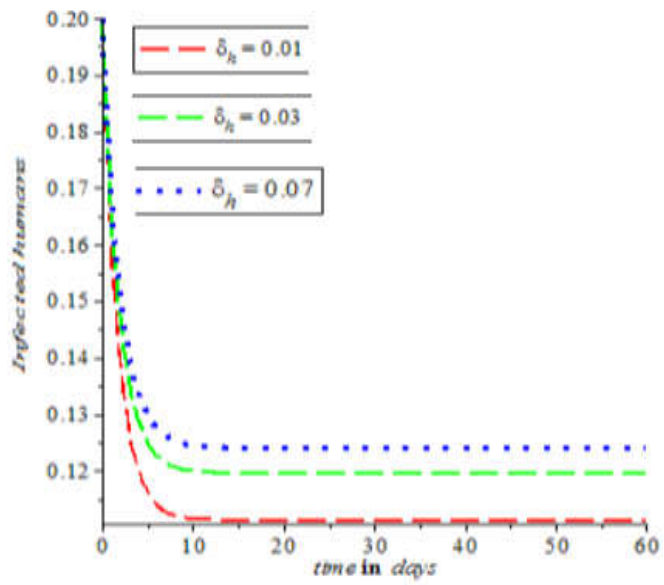

Fig. 1 Graph of infected humans against time with different rate of human treatment

Conclusion: In this study, the population was divided into susceptible, infected and recovered classes. A model with two control strategies was developed one for human and the other one for insecticide vectors. The basic reproduction number is calculated to know whether the disease will persist or die out with the help of global stability and endemic state. Surveillance strategy should put in place by the entire societal to combat the disease at early stage. Taking into account, prevention and quarantine was not incorporated due to time limit for further study.

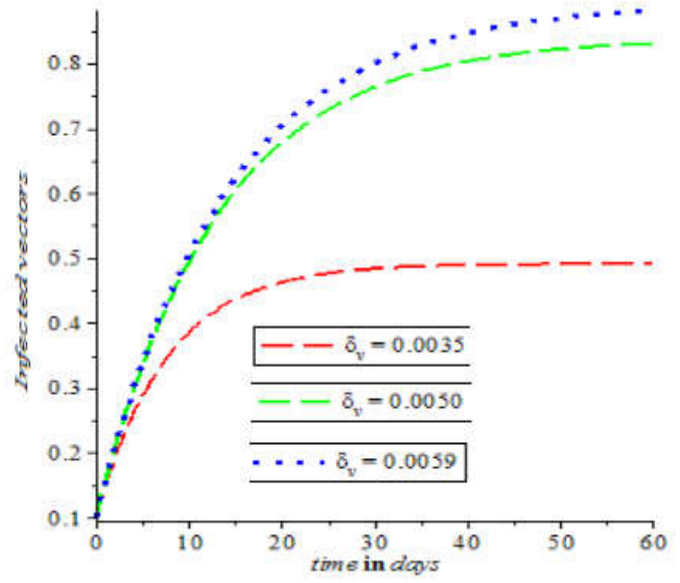

Fig. 2 Graph of infected vectors against time with different rate of insecticides on vectors

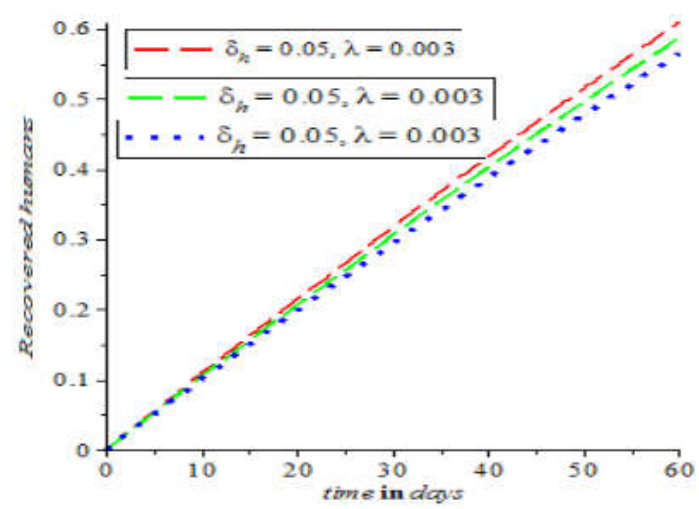

Fig. 3 Graph of recovered humans against time with different rates of humans treatment and recovered respectively

\section{REFERENCES}

Adamu, G; Bawa, M; Jiya, M; Chado, U. C (2017). A Mathematical Model for the Dynamics of Zika Virus via Homotopy Perturbation Method: J. Appl. Sci. Environ. Manage. 21(4), 615-623.

Adam, J. K; Sebastian, F; Rosalind, M. E; HenriPierre, M. W; John, E; Eric, J. $\mathrm{N}(2014)$. Transmission dynamics of Zika virus in island populations: A Modelling Analysis of the French Polynesia outbreak. 1-15.

Cooke, K. L; Van Den Driessche, P (1996). Analysis of an SEIRS epidemic model withtwo delays. Journal of Mathematical Biology, 35(2), 240-260.

Diagne, C. T; Diallo, D; Faye, O; Ba, Y; Faye, O; Gaye, A; Dia, I; Weaver, S. C; Sall, A.A; Diallo, $M$ (2015). Potential of selected Senegalese Aedesspp, mosquitoes Diptera: Culicidaeto 
transmit Zika virus. BMC Infect Dis. 15(492), 12312 .

Diallo, D; Sall, A. A; Diagne, C. T; Faye, O; Ba, Y; Hanley, K. A; Buenemann, M; Weaver, S. C; Diallo, $M$ (2014). Zika virus emergence in mosquitoes in south eastern Senegal. 9(10).

Dick, G. W (1952). Zika virus. II. Pathogenicity and physical properties. Trans R Soc Trop. Med Hyg. 46(5), 521-534.

Dick, G. W; Kitchen, S. F; Haddow, A. J (1952). Zika virus. I. Isolations and serological specificity. Trans R Soc Trop Med Hyg, 46 (5), 509-520.

Ebenezer, O; Kazeem, O. O (2016). Mathematical Modelling of Zika virus. Asian PacificJournal of Tropical Disease, 6(9), 673-679.

Gao, D; Lou, Y; He, D; Porco, T. C; Kuang, Y; Chowell, G; Ruan, S (2016). Prevention and control of Zika as a mosquito-borne and sexually transmitted disease: a mathematical modelling analysis. Scientific reports, 6, 28070.

Haddow, A. J; Williams, M. C; Woodall, J.P; Simpson, D.I; Goma, L. K (1964). Twelve isolations of Zika virus from Aedes (Stegomyia) africanus (Theobald) taken in and above a Uganda forest. Bull World Health Organ31:57-69.

Jiraporn, L; Puntani, P (2017). The Zika virus Transmission model: International Journal of Bioscience, Biochemistry and Bioinformatics. $7(2), 66-73$

McCrae, A. W; Kirya, B. G (1982). Yellow fever and Zika virus epizootics and enzooticsin Uganda. Trans R Soc Trop Med Hyg. 76(4), 552-562.

Mlakar, J; Korva, M; Tul, N; Popović, M; PoljšakPrijatelj, M; Mraz, J; Kolenc, M; Resman, R. K; Vesnaver, V. T; Fabjan, V. V; Vizjak, A; Pižem, J; Petrovec, M; Avšič, Ž. T (2016). Zika virus associated with microcephaly. N. Engl. J. Med 374 (10), 951-958.
Mpeshe, S. C; Nkuba, N; Stephano, S (2017). Modelling Approach to Investigate the Dynamics of Zika virus fever: A neglected Disease in Africa. International Journal of Advances in Applied Mathematics and Mechanics, 4(3), 14-21.

Musso, D; Roche, C; Robin, E; Nhan, T; Teissier, A; Cao-Lormeau, V. M (2014).Potential Sexual Transmission of Zika Virus. Emerging infectious diseases, 21(2), 359.

Musso, D (2015). Zika virus transmission from French Polynesia to Brazil. EmergInfectDis, 21(10), 1887.

Okongo, M. O (2015). The local and global stability of the disease free equilibrium in a co- infection model of HIV/AIDS, tubercolosis and malaria. IOSR J. Math, 11, 33-43.

Oliveira, M. A. S; Malinger, G; Ximenes, R; Szejnfeld, P. O; Alves, S. S; Bispo de, F.A. M (2016). Zika virus intrauterine infection causes fetal brain abnormality andmicrocephaly: tip of the iceberg. Ultrasound Obstet. Gynecol. 47(1), 6-7

Onuorah, M. O; Ademu, A; Obi, E. I; Hasheem, A. M (2016). Deterministic Model ofZika Virus. Research Journal of Mathematics, 3(3), 1-15.

Van den Driessche, P (2017). Reproduction numbers of infectious disease models. Infectious Disease Modelling, 2(3), 288-303.
WHO
(2016)
Retrieved from virushhttp://www.who.int/mediacentre/factsheets /zika/en, (22 May 2018). 